\title{
Effect of Different Splint Thicknesses on Occlusal Function and Temporomandibular Joint Sounds: A Clinical Report
}

\author{
Yuyao Tang*, Hefei Li*, Yuming Chen, Lisha Zhu, Hong Kang\# \\ Department of Prosthodontics, Lanzhou University School of Stomatology, Lanzhou, China \\ Email: tangyy2016@lzu.edu.cn, lihf14@lzu.edu.cn, ymchen14@lzu.edu.cn, zhulsh14@lzu.edu.cn, "kanghong@lzu.edu.cn
}

How to cite this paper: Tang, Y.Y., Li, H.F., Chen, Y.M., Zhu, L.S. and Kang, H. (2018) Effect of Different Splint Thicknesses on Occlusal Function and Temporomandibular Joint Sounds: A Clinical Report. Open Journal of Stomatology, 8, 326-337.

https://doi.org/10.4236/ojst.2018.812031

Received: December 29, 2017

Accepted: December 9, 2018

Published: December 12, 2018

Copyright $\odot 2018$ by authors and Scientific Research Publishing Inc. This work is licensed under the Creative Commons Attribution International License (CC BY 4.0).

http://creativecommons.org/licenses/by/4.0/

\begin{abstract}
Background: Occlusal splint therapy, which is a conservative approach to treat temporomandibular disorders (TMDs) and bruxism, can change the occlusal contact of dentition. However, little is known about the variation in bite force and temporomandibular joint (TMJ) sounds. The objective of this case report is to compare the effects of different splint thicknesses constructed by vacuum lamination technology on occlusal function and TMJ sounds. Clinical Presentation: This study presents a 24-year-old male with bruxism. Four splints of different thicknesses $(0.6 \mathrm{~mm}, 1 \mathrm{~mm}, 2.5 \mathrm{~mm}$ and 3 $\mathrm{mm}$ ) were fabricated and tested. Then, an array of occlusal data were recorded and analyzed by the T-Scan ${ }^{\circledR}$ III system and joint vibration analysis (JVA). Conclusion: The results reveal that splints of different thicknesses manufactured by the vacuum hot-lamination apparatus may cause a change in occlusal force.
\end{abstract}

\section{Keywords}

Bruxism, Splint, T-Scan ${ }^{\circledR}$ III, JVA, Bite Force

\section{Introduction}

Sleep bruxism (SB) is defined as a stereotyped movement disorder associated with involuntary jaw muscle activity. SB is characterized by occasional grinding of the teeth or by bracing of the mandible during sleep and is often accompanied by oral facial pain or fatigue in the morning [1]. SB can be an important etiological factor of abnormal tooth wear, destruction of dental restoration, periodontal diseases and TMDs.

${ }^{\star}$ These authors have contributed equally to this paper. 
The splint was first introduced in 1901 by Karolyi [2] for the treatment of bruxism. Hence, the use of occlusal splint soon became a noninvasive option for treating TMD and bruxism due to its simplicity and reversibility. The splint can occupy a certain amount of space in its thickness and provides a bio-lever fulcrum to induce a change in the mandibular position and achieve the intended therapeutic fit [3]. With the recent development of vacuum lamination technology, soft, resilient splint therapy has been increasingly advocated to treat patients with SB and to reduce masticatory muscle hyperactivity and maxillofacial pain [4]. Furthermore, even when used for an extended period of time, splints do not lead to a change in the occlusal relationship [5].

The therapeutic properties of splints have been discussed previously in the literature in regard to their use as static indicator, which demonstrates their effectiveness in treating TMD and bruxism. Clinicians use splints to produce dental contacts and to obtain new occlusal equilibrium and functional integrity [6]. However, only a limited number of studies have attempted to evaluate the effects of different splint thicknesses on bite force and temporomandibular joint (TMJ) sounds.

Bite force is considered an important indicator of masticatory function [5] and is often measured as the maximum bite force (MBF), which is exerted by the elevator muscles and is regulated by the nervous, muscular, skeletal and dental systems. The factors associated with MBF include malocclusion, occlusal contact area, body size, interocclusal separations, the location of the measuring device on the dentition, the posture of the subject's head at the time of measurement, age, height, weight and sexual dimorphism [7] [8] [9].

Maness first introduced the T-scan III system at the end of the 1980s [10]. The T-Scan ${ }^{\circledR}$ III digital occlusal force analysis system (Bimetallic Co., T-Scan ${ }^{\circledR} \mathrm{III}$ V8.01, USA) includes a USB handle, processing unit, $1 \mathrm{~mm}$-thickness sensor, and T-Scan ${ }^{\circledR}$ III software, which can record the occlusal contact sequence in real time [11]. When a patient intercuspates, the resistance of the contact points of the sensor changes due to the stress, and the output of the sensor can be displayed graphically within two dental arches and in two dimensions to show where occlusal contact occurs. The upper and lower arches shown on the screen are divided into four parts: the left-anterior quadrant, left-posterior quadrant, right-anterior quadrant and right-posterior quadrant. The parameters of the T-Scan ${ }^{\circledR} \mathrm{III}$ system include the occlusal center, occlusal force in each tooth, occlusal force distribution in the anterior and posterior quadrants and the bilateral (right-left) force distribution [11]. M. Cerna used the T-Scan ${ }^{\circledR}$ III system to record the distribution of bite force, and the results demonstrated the validity and reliability of this electronic digital system [10].

Sounds from the TMJ are one of the most frequently reported symptoms of TMDs [12]. JVA is based on electrovibratography using the Bio-PAK system (Bioresearch Inc., Milwaukee, USA) and is an advanced technique developed to record TMJ sounds. Using this system, it is possible to identify the vibrations that 
are produced by joint tissues during opening and closing movements, distinguish various wave patterns, analyze the eventual vibrations in the contralateral condyle, and calculate the frequency and amplitude of the vibration, which allows clinicians to determine whether quantifiable changes have occurred in the TMJ of bruxism patients. In a previous study, In-Taek Hwang used the Bio-PAK system to analyze the joint tone spectrum and recommended that the ratio of the integral of sounds $>300 \mathrm{~Hz} /<300 \mathrm{~Hz}$ and the frequency spectrum may be two important factors for patient diagnosis [13].

\section{Case Report}

The subject was a 24-year-old male with SB, no missing teeth, edentulous space in the arches, no malocclusion, and removable dentures and orthodontic appliances in his mouth. A maxillary impression of the patient was made with polyvinyl siloxane (ImpregumF; 3 M ESPE, St. Paul, Minn), and a cast was obtained using mixed Type III Dental gypsum. Then, the upper cast was placed into avacuum hot-lamination apparatus (GER, EKRODENT) to fabricate four different splint thicknesses (Figure 1). The experimental condition consisted of a series of resin splints of different thicknesses $(0.6,1.0,2.5$ and $3 \mathrm{~mm})$, and the control condition consisted of no splint.

\subsection{Bite Force}

To initialize the T-Scan ${ }^{\circledR}$ III software, the width of the maxillary first incisors of the individual were measured using a Vernier caliper. The participant was asked to wear the occlusal splint, and a recording sensor was placed beneath the upper teeth. The participant intercuspated with maximum occlusal force for 3 seconds before repeating the same process twice followed by a 3-minute interval to avoid muscular fatigue. The bite force reading on the charged meter was then recorded (Figure 2). The dynamic process was then repeated for each splint.

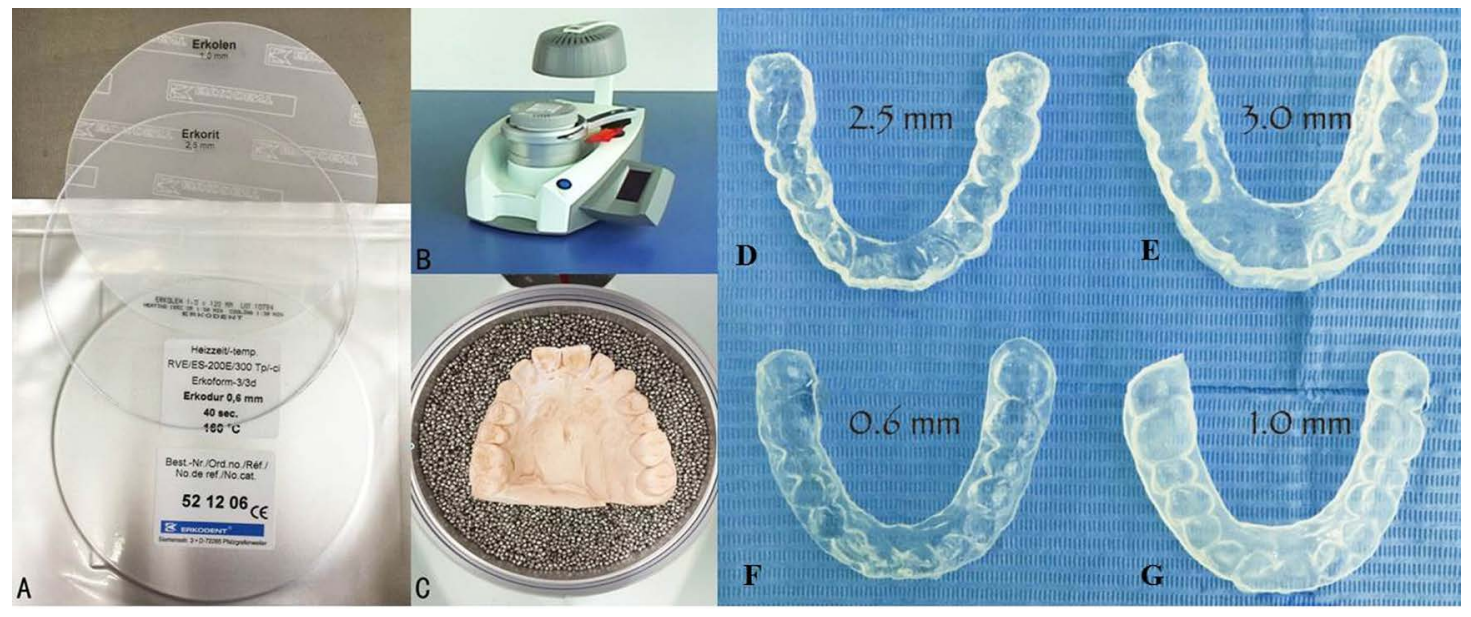

Figure 1. Producing different thickness of splints. (A) Plate materials; (B) Vacuum hot-lamination apparatus; (C) The cast was placed beneath of vacuum machine, preparing the splints; (D) $2.5 \mathrm{~mm}$ thickness hard-splint;

(E) $3 \mathrm{~mm}$ thickness soft-splint; (F) $0.6 \mathrm{~mm}$ thickness hard-splint; (G) $1 \mathrm{~mm}$ thickness hard-splint. 


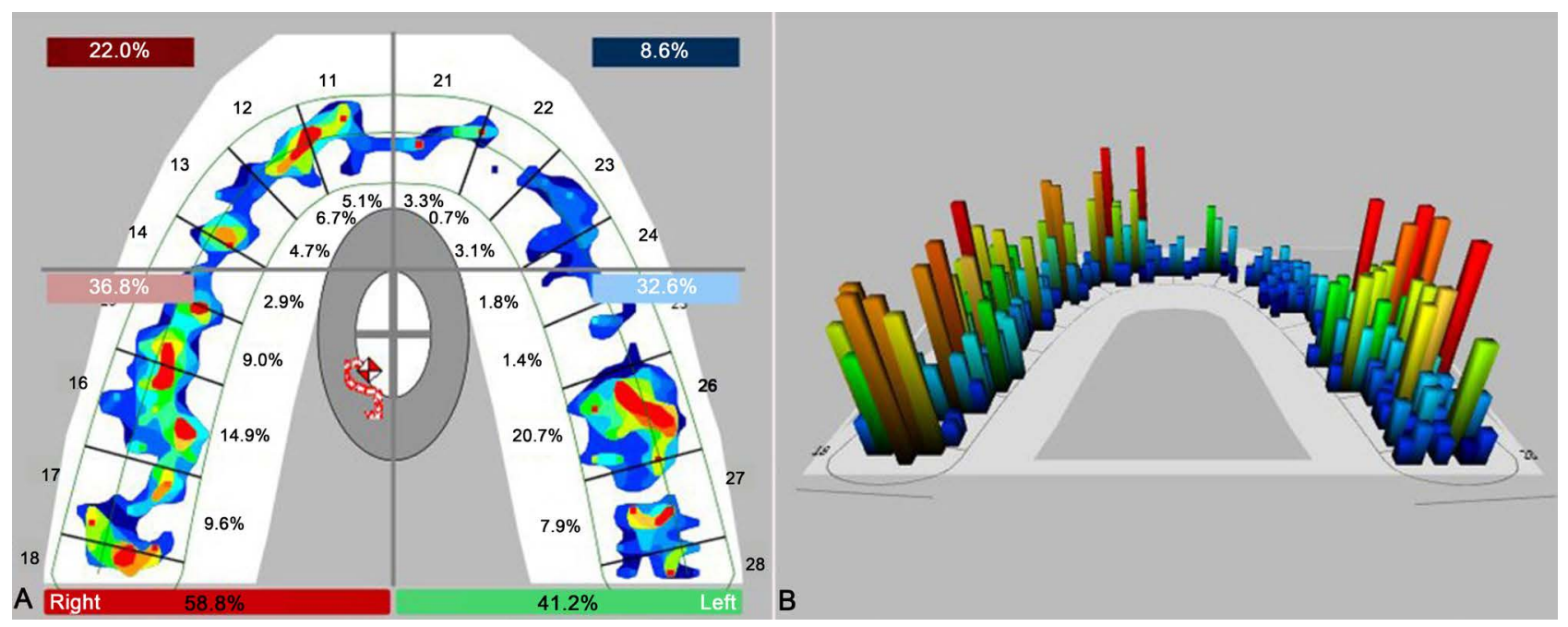

Figure 2. This software divided the arch into four areas, including left-anterior, right-anterior, left-posterior and right-posterior quadrants. The anterior part consisted of three anterior teeth (central incisor, lateral incisor, and canine), while premolar and molar teeth are included in the both posterior quadrants.

When the bite force reached $25 \%, 50 \%, 75 \%$, and $100 \%$ of the MBF, the data were collected and calculated as follows:

- Bilateral force distribution between the left and right sides of the arch.

- The location of the bite force center.

- Anterior and posterior bite force distribution.

- Asymmetry index of the bite force.

\subsection{Temporomandibular Joint Sounds}

During the process of data collection, the subject sat comfortably on a dental chair in an upright position while the method of TMJ sound measurement was explained. The joint vibration was detected using stereophonic headphones connected to a corresponding input transmission device. When the patient initiated the opening and closing mouth movement, an animation was shown on the screen at a moderate speed, the headphones placed over the particular area received the vibration, and the software simultaneously transformed the data into wave forms. The total integral of the vibration energy, ratio of the integral between frequencies above $300 \mathrm{~Hz}$ and below $300 \mathrm{~Hz}(>300 \mathrm{~Hz} /<300 \mathrm{~Hz}$ ratio), peak frequency, median frequency, peak amplitude and distance to the centric occlusion position were recorded.

\subsection{Data Analysis and Results}

The distribution of the occlusal force was displayed in each quadrant and was also tabulated in an Excel spreadsheet. To compare the in-group differences, a paired Student's t-test was performed using IBM SPSS statistics version 22.0 (IBM Corp., Armonk, NY, USA). In addition, the Dowell ruler (Dowell, V1.0.0.110. China) was applied to measure the location coordinates of the bite force center. A difference resulting in a value of $\alpha=0.05$ was considered significant. 
The occlusal force distributions of the left and right side after wearing the splints are shown in Table 1 , and the center of the bite force is shown in Figure 3. The asymmetry indexes of the different conditions are shown in Table 2. The proportion of the total anterior tooth bite force is shown in Table 3. The integrals of the $>300 \mathrm{~Hz} /<300 \mathrm{~Hz}$ ratios in the different conditions are shown in Table 4, and the TMJ sounds in the different conditions are shown in Figure 4. We then compared the experimental conditions with the control condition.

Table 1. The bite force percentage in the left and right sides of different groups (average \pm standard deviation).

\begin{tabular}{|c|c|c|c|c|c|c|c|c|}
\hline Splint thickness & \multicolumn{2}{|c|}{$25 \% \mathrm{MBF}$} & \multicolumn{2}{|c|}{$50 \% \mathrm{MBF}$} & \multicolumn{2}{|c|}{$75 \% \mathrm{MBF}$} & \multicolumn{2}{|c|}{$100 \% \mathrm{MBF}$} \\
\hline $0 \mathrm{~mm}$ & $39.80 \pm 2.92$ & $60.20 \pm 2.92$ & $45.03 \pm 2.70$ & $54.967 \pm 2.70$ & $49.30 \pm 3.72$ & $50.70 \pm 3.72$ & $51.93 \pm 0.83$ & $48.07 \pm 0.83$ \\
\hline $1 \mathrm{~mm}$ & $39.15 \pm 0.78$ & $60.85 \pm 0.78$ & $42.53 \pm 3.69$ & $57.47 \pm 3.69$ & $44.83 \pm 3.07$ & $55.17 \pm 3.07$ & $47.27 \pm 0.71$ & $52.73 \pm 0.71$ \\
\hline $2.5 \mathrm{~mm}$ & $50.40 \pm 5.09$ & $49.60 \pm 5.09$ & $52.15 \pm 1.77$ & $47.85 \pm 1.77$ & $47.20 \pm 14.84$ & $52.80 \pm 14.84$ & $52.07 \pm 11.26$ & $47.93 \pm 11.26$ \\
\hline $3 \mathrm{~mm}$ & $49.93 \pm 2.46$ & $50.07 \pm 2.46$ & $49.33 \pm 1.65$ & $50.67 \pm 1.65$ & $48.50 \pm 1.13$ & $51.50 \pm 1.13$ & $49.00 \pm 0.17$ & $51.03 \pm 0.15$ \\
\hline $\mathrm{p}$ & 0.016 & 0.014 & 0.002 & 0.001 & 0.503 & 0.503 & 0.152 & 0.152 \\
\hline
\end{tabular}

*MBF: Maximum bite force.

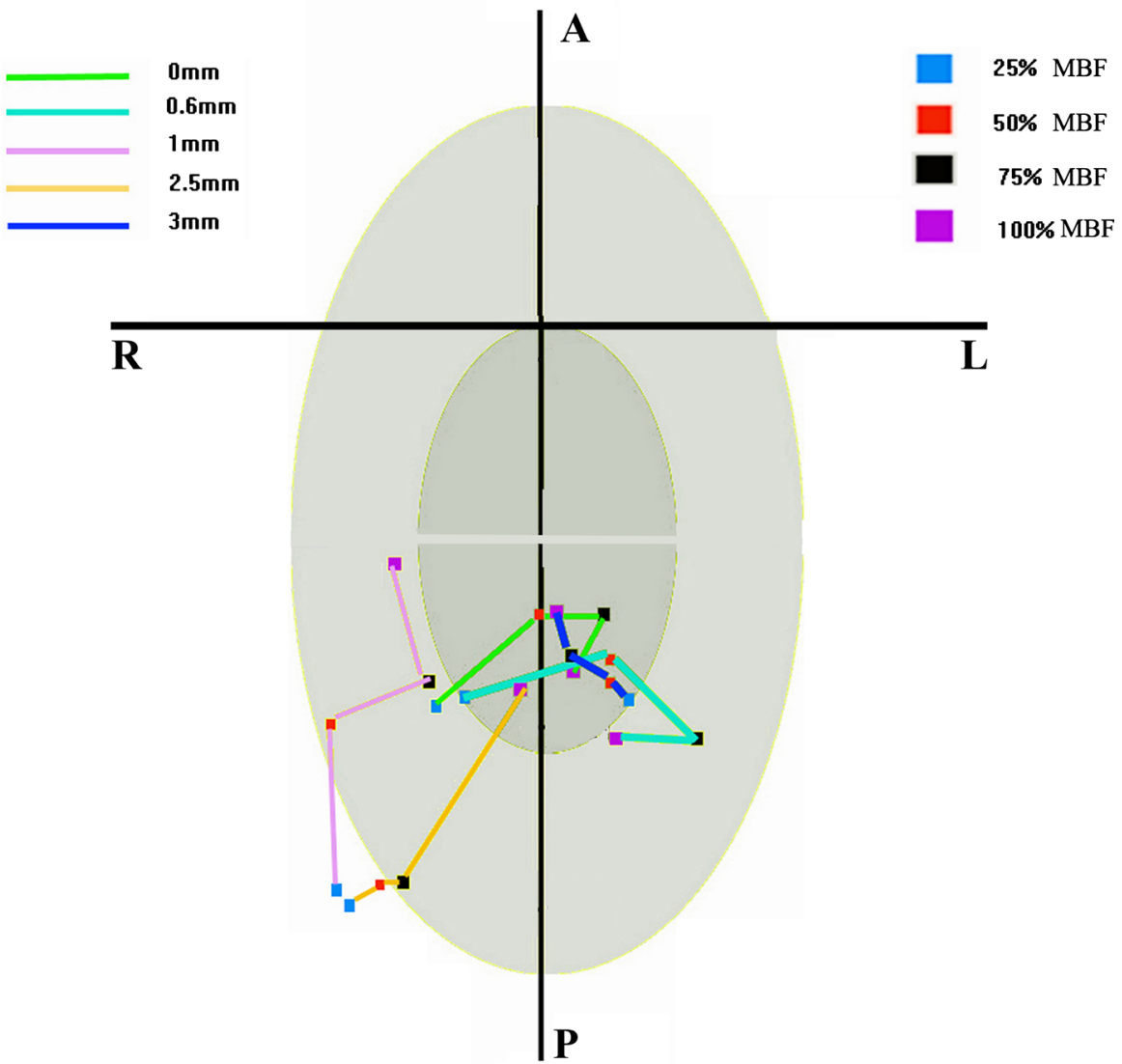

*A.Anterior P.Posterior L. Left R. Right

Figure 3. The track of occlusal centre (the gray oval and black cross imitated the interface of T-Scan ${ }^{\circledR}$ III system). 
Table 2. The asymmetry index of the bite force in different groups (average \pm standard deviation).

\begin{tabular}{ccccc}
\hline Splint thickness & $25 \% \mathrm{MBF}$ & $50 \% \mathrm{MBF}$ & $75 \% \mathrm{MBF}$ & $100 \% \mathrm{MBF}$ \\
\hline $0 \mathrm{~mm}$ & $20.40 \pm 5.84$ & $9.93 \pm 5.39$ & $5.13 \pm 4.35$ & $3.87 \pm 1.67$ \\
$0.6 \mathrm{~mm}$ & $17.80 \pm 1.40$ & $10.13 \pm 4.41$ & $7.53 \pm 10.49$ & $12.40 \pm 6.063$ \\
$1 \mathrm{~mm}$ & $21.70 \pm 1.56$ & $14.93 \pm 7.38$ & $10.33 \pm 6.15$ & $5.47 \pm 1.42$ \\
$2.5 \mathrm{~mm}$ & $7.20 \pm 1.13$ & $4.30 \pm 3.54$ & $20.80 \pm 16.72$ & $18.40 \pm 5.02$ \\
$3 \mathrm{~mm}$ & $7.20 \pm 1.81$ & $2.27 \pm 2.42$ & $3.00 \pm 2.25$ & $2.00 \pm 0.35$ \\
$\mathrm{p}$ & 0.002 & 0.001 & 0.076 & 0.000 \\
\hline
\end{tabular}

${ }^{\star}$ MBF: Maximum bite force.

Table 3. The proportion of the anterior tooth bite force in different groups (average \pm standard deviation).

\begin{tabular}{ccccc}
\hline Splint thickness & $25 \% \mathrm{MBF}$ & $50 \% \mathrm{MBF}$ & $75 \% \mathrm{MBF}$ & $100 \% \mathrm{MBF}$ \\
\hline $0 \mathrm{~mm}$ & $31.77 \pm 1.75$ & $33.37 \pm 1.20$ & $31.57 \pm 0.32$ & $29.67 \pm 1.27$ \\
$0.6 \mathrm{~mm}$ & $23.00 \pm 1.35$ & $24.27 \pm 2.200$ & $22.43 \pm 1.40$ & $21.20 \pm 1.42$ \\
$1 \mathrm{~mm}$ & $9.75 \pm 2.05$ & $12.13 \pm 2.32$ & $13.07 \pm 2.200$ & $13.40 \pm 1.65$ \\
$2.5 \mathrm{~mm}$ & $25.25 \pm 6.29$ & $29.65 \pm 8.41$ & $30.73 \pm 5.22$ & $28.67 \pm 5.10$ \\
$3 \mathrm{~mm}$ & $4.07 \pm 1.40$ & $11.17 \pm 1.36$ & $19.73 \pm 0.64$ & $28.07 \pm 0.96$ \\
$\mathrm{p}$ & 0.000 & 0.000 & 0.000 & 0.000 \\
\hline
\end{tabular}

${ }^{*}$ MBF: Maximum bite force.

Table 4. Integral > 300/ < 300 Ratios in different groups.

\begin{tabular}{|c|c|c|c|c|c|c|c|c|c|c|}
\hline \multirow{2}{*}{$\begin{array}{c}\text { Splint } \\
\text { thickness }\end{array}$} & \multicolumn{2}{|c|}{$0 \mathrm{~mm}$} & \multicolumn{2}{|c|}{$0.6 \mathrm{~mm}$} & \multicolumn{2}{|c|}{$1 \mathrm{~mm}$} & \multicolumn{2}{|c|}{$2.5 \mathrm{~mm}$} & \multicolumn{2}{|c|}{$3 \mathrm{~mm}$} \\
\hline & Lt. Ratio & Rt. Ratio & Lt. Ratio & Rt. Ratio & Lt. Ratio & Rt. Ratio & Lt. Ratio & Rt. Ratio & Lt. Ratio & Rt. Ratio \\
\hline & 1.6 & 0.7 & 0.05 & 0.09 & 0.06 & 0.12 & 0.08 & 0.15 & 0.07 & 0.10 \\
\hline
\end{tabular}

When $25 \%$ and $50 \%$ of the maximum occlusal force was achieved, significant differences were found for the $2.5 \mathrm{~mm}$ and $3 \mathrm{~mm}$ splint $(\mathrm{p}<0.05)$ compared to the control condition (according to the index of occlusal force distribution in the left and right sides). By contrast, at $75 \%$ and $100 \%$ of the MBF, no significant differences were found for either the $2.5 \mathrm{~mm}$ or $3 \mathrm{~mm}$ splint compared to the control condition $(p>0.05)$. Regarding the $2.5 \mathrm{~mm}$ splint, the location of the occlusal center was mostly in the gray ellipse at $100 \%$ MBF $(p<0.05)$. The asymmetry indexes of the $3 \mathrm{~mm}$ splint at $25 \%, 50 \%$, and $100 \% \mathrm{MBF}$ were higher than those in the control condition $(\mathrm{p}<0.05)$. The proportions of the anterior tooth occlusal force for the $2.5 \mathrm{~mm}$ splint at $25 \%, 50 \%, 75 \%$, and $100 \%$ MBF were consistent with those of the control condition $(\mathrm{p}<0.01)$. The integrals of the $>300$ $\mathrm{Hz} /<300 \mathrm{~Hz}$ ratio were significantly different $(\mathrm{p}<0.05)$ for all four splint treatments compared to the control condition. A tendency toward smooth joint waveforms was observed with increasing thickness, especially for the right side. 

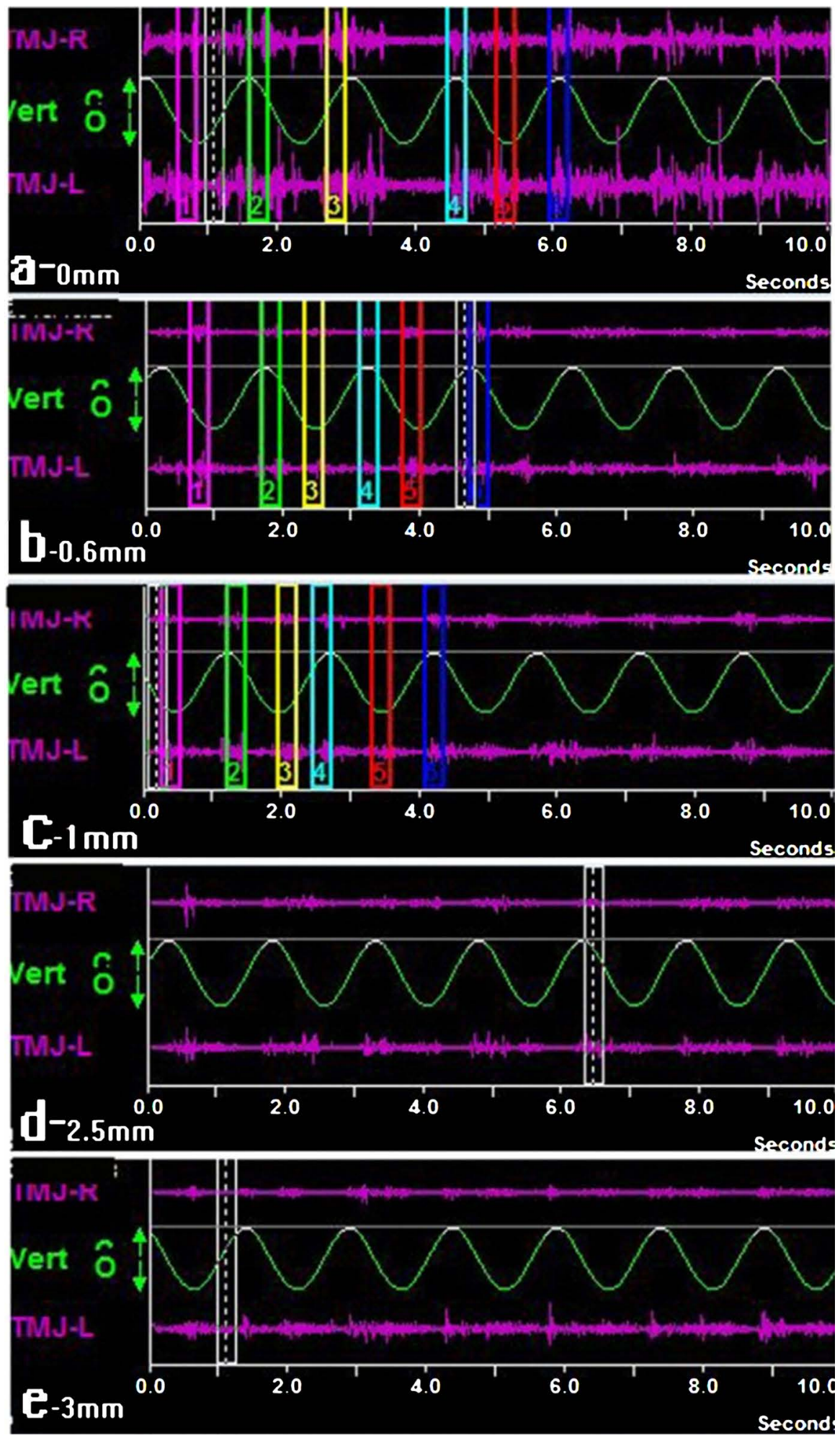

Figure 4. Temporomandibular joint sound waves in different groups.

\section{Discussion}

Splints have been used for treatment of the stomatognathic system for more than 100 years. The current literature shows that the most commonly used splints are 
the soft resilient splint and the stabilization splint, which will change the original relationship between the oral contact points and the TMJ [14] [15] [16]. Consequently, this change will disrupt the original memory type of the masticatory muscle [9]. This study compared occlusal force distribution percentages measured by T-scans between experimental conditions and the control condition.

Analysis of the results revealed that the bite force on the right side was significantly greater than that on the left side at $25 \%$ and $50 \% \mathrm{MBF}$ when the subject did not wear a splint. However, the subject's bilateral bite force tended to be balanced with increasing splint thickness $(\mathrm{p}<0.05)$. No significant difference was observed between the left and right side for either the experimental or control conditions at $75 \%$ and $100 \%$ of the MBF. This may be correlated with the patient's unhealthy habit of unilateral mastication. After wearing different splints, the bite force distribution of the anterior teeth was reduced compared to the posterior teeth, whereas the $2.5 \mathrm{~mm}$-thick splint was an exception; in this condition, the anterior teeth remained the main area of support.

According to the T-Scan ${ }^{\circledR}$ III system, the occlusal force center is the balance center of all oral contact points [17]. The center is located in the premolar area in healthy individuals, which is defined in the elliptical circle that is close to the midline [18]. In this case, the center points of the $3 \mathrm{~mm}$ and $0.6 \mathrm{~mm}$ splints were located in the elliptical circle and were relatively consistent with the control condition. The results reported in the clinical study of H. Kurita [19] indicated that for patients with masticatory muscle disorders who wore stabilization splints for 4 weeks, the integrated occlusal load was normalized. According to Senay Canay, after muscle relaxation splint therapy, EMG activity was increased in the left and right temporal muscles [20]. Splint therapy can compensate for an abnormal occlusal force center, which reduces the incidence of early contact and eventually leads to a change inocclusal contact in TMD patients.

The asymmetric index represents the balance of the left and right bite forces [16] (Left-right asymmetry $=$ (total right occlusal force - total left occlusal force)/(total right occlusal force + total left occlusal force) $\times 100 \%$ ). A smaller difference in bilateral bite force results in improved balance of both sides. In this case, significantly symmetrical changes occurred on the right and left side after wearing a $3 \mathrm{~mm}$ splint, whereas other splints showed no sign of improvement. Senay Canay's studies reported no significant differences regarding the asymmetry index before and after two weeks and four weeks of splint therapy [20], which may be related to the full adaption of the masticatory muscles to the existing occlusal relationship after long-term splint use.

The ratio of the bite force of the anterior teeth represents the percentage of the anterior teeth in the full bite force [16]. Based on the periodontal membrane area of the anterior and posterior teeth, it is generally believed that the bite force ratio between the anterior and posterior teeth is 3:7. In this case, the values of the experimental conditions with the $2.5 \mathrm{~mm}$ and $0.6 \mathrm{~mm}$ splints were consistent with those of the control condition. The statistical analysis show a slight difference be- 
tween the condition with no splint and the $2.5 \mathrm{~mm}$ and $0.6 \mathrm{~mm}$ splint conditions.

Our findings for the TMJ sounds suggested that all splints (regardless of thickness) decreased the peak amplitudes. However, the peak amplitudes were milder with increasing splint thickness, while symptomatic TMJ vibrations were mild [21]. The joint waveforms tended to be stable and showed less fluctuation with increasing splint thickness, especially on the right side, which indicated that changing the thickness of the splint benefited the TMJ of patients with bruxism, which may provide a reference for clinical treatment.

Several studies have shown that short-term interocclusal appliance therapy had a positive effect on bite force in SB patients [22] [23]. In Duygu Karakis's clinical test [24], patients were asked to wear a canine protected hard stabilization splint $(2 \mathrm{~mm})$ and a Bruxo Guard soft splint $(2 \mathrm{~mm})$ during sleep for 6 weeks. The results from the canine protected hard stabilization splint group indicated a significant increase in maximum occlusal force. The clinical symptoms of the participants who used the Bruxo Guard soft splint improved; however, the MBF decreased. The splint did not reduce the activity of SB in long-term observations [25] [26]. Patients showed a more than 50\% reduction of SB activity while wearing a stabilization splint [27] [28] [29]. A study by T. Harda found that wearing a stabilization splint had an immediate effect on muscle activity, but the amplitude of masseter muscle activity on electromyography was reduced after 2 weeks, 4 weeks and 6 weeks. Briefly, the stabilization splint had a short-term impact on the masseter muscle [23]. In this case, data were collected as soon as the patient began wearing the splints. To improve the integrity and reliability of the retrospective data, examination of the long-term effects of splints should be performed, including data collection after several weeks (one week, two weeks, one month) of wearing the splints. In future experiments, the aim will be to increase the sample size and further explore the effect of splints on bite force.

\section{Conclusion}

The maximum bite forces of the left and right sides were not significantly different between the experimental and control conditions. The center points of the $3 \mathrm{~mm}$ and $0.6 \mathrm{~mm}$ splints were located in the elliptical circle and were relatively consistent with those of the control condition. For the $3 \mathrm{~mm}$ splint, all indicators used to measure occlusal force except the proportion of the anterior tooth bite force presented improved tendencies compared to the control condition and the other splints. Although not statistically confirmed, similar ratios of the anterior and posterior teeth were observed when the patient wore the $2.5 \mathrm{~mm}$ and 0.6 $\mathrm{mm}$ splints, and the ratio was close to 3:7. Using vacuum lamination technology, splints of different thicknesses can be produced that may result in a change of the occlusal force.

\section{Acknowledgements}

This work was supported by the National Innovation and Entrepreneurship Ac- 
tion Plan Project of Lanzhou University (no. 201710730159).

\section{Conflicts of interest}

The authors declare no conflicts of interest regarding the publication of this paper.

\section{References}

[1] Thymi, M., Visscher, C.M., Yoshida-Kohno, E., et al. (2017) Associations between Sleep Bruxism and (Peri-)implant Complications: A Prospective Cohort Study. BDJ Open, 3, Article ID: 17003. https://doi.org/10.1038/bdjopen.2017.3

[2] Ramfjord, S.P. and Ash, M.M. (1994) Reflection on the Michigan Occlusal Splint. Journal of Oral Rehabilitation, 21, 491-500. https://doi.org/10.1111/j.1365-2842.1994.tb01164.x

[3] Yi, X.Z. (2003) Third Edition of textbook of Occlusion. People's Medical Publishing House, Beijing, 160-161. (In Chinese)

[4] Thanathornwong, B. and Suebnukarn, S. (2017) Clinical Decision Support Model to Predict Occlusal Force in Bruxism Patients. Healthcare Informatics Research, 23, 255-261. https://doi.org/10.4258/hir.2017.23.4.255

[5] Naikmasur, V., Bhargava, P., Guttal, K., et al. (2008) Soft Occlusal Splint Therapy in the Management of Myofascial Pain Dysfunction Syndrome: A Follow-Up Study. Indian Journal of Dental Research, 19, 196-203.

https://doi.org/10.4103/0970-9290.42950

[6] Cohen-Levy, J. (2015) Orthodontic T-Scan Applications. In: Kerstein, R.B., Ed., Handbook of Research: Computerized Occlusal Analysis Technology Applications in Dental Medicine, Vol.2, IGI Global. Hershey, PA, 523-561.

https://doi.org/10.4018/978-1-4666-6587-3.ch011

[7] Pereira-Cenci, T., Pereira, L.J., Cenci, M.S., et al. (2007) Maximal Bite Force and Its Association with Temporomandibular Disorders. Brazilian Dental Journal, 18, 65-68. https://doi.org/10.1590/S0103-64402007000100014

[8] Tortopidis, D., Lyons, M.F., Baxendale, R.H., et al. (1998) The Variability of Bite Force Measurement between Sessions, in Different Positions within the Dental Arch. Journal of Oral Rehabilitation, 25, 681-686.

https://doi.org/10.1046/j.1365-2842.1998.00293.x

[9] Koc, D., Dogan, A. and Bek, B. (2010) Biteforce and Influential Factors on Bite Force Measurements: A Literature Review. European Journal of Dentistry, 4, 223-232.

[10] Cerna, M., Ferreira, R., Zaror, C., et al. (2015) Validity and Reliability of the T-Scan $\left({ }^{(}\right)$III for Measuring Force Under Laboratory Conditions. Journal of Oral Rehabilitation, 42, 544-551. https://doi.org/10.1111/joor.12284

[11] Qadeer, S., Yang, L., Sarinnaphakorn, L., et al. (2016) Comparison of Closure Occlusal Force Parameters in Post-Orthodontic and Non-Orthodontic Subjects Using T-Scan ${ }^{\circledR}$ III DMD Occlusal Analysis. Journal of Craniomandibular \& Sleep Practice, 34, 395-401.

[12] Zhang, J., Whittle, T., Wang, L., et al. (2014) The Reproducibility of Temporomandibular Joint Vibrations over Time in the Human. Journal of Oral Rehabilitation, 41, 206-217. https://doi.org/10.1111/joor.12141

[13] Hwang, I.T., Jung, D.U., Lee, J.H., et al. (2009) Evaluation of TMJ Sound on the 
Subject with TMJ Disorder by Joint Vibration Analysis. Journal of Advanced Prosthodontics, 1 26-30. https://doi.org/10.4047/jap.2009.1.1.26

[14] Kang, H. and Wang, J.W. (2011) Treatment of Stomatognathic Dysfunction with Occlusal Splints. Chinese Journal of Practicalstomatology, 76, 318-327. (In Chinese)

[15] Aksakalli, S., Temucin, F., Pamukcu, A., et al. (2015) Effectiveness of Two Different Splints to Treat Temporomandibular Disorders. Journal of Orofacial Orthopedics, 76, 318-327. https://doi.org/10.1007/s00056-015-0294-4

[16] Conti, P.C., Dos Santos, C.N., Kogawa, E.M., et al. (2006) The Treatment of Painful Temporomandibular Joint Clicking with Oral Splints: A Randomized Clinical Trial. Journal of the American Dental Association, 137, 1108-1114. https://doi.org/10.14219/jada.archive.2006.0349

[17] Liu, C.W., Chang, Y.M., Shen, Y.F., et al. (2015) Using the T-Scan ${ }^{\circledR}$ III System to Analyze Occlusal Function in Mandibular Reconstruction Patients: A Pilot Study. Biomedical Journal, 38, 52-57.

[18] Cheng, H.J., Geng, Y. and Zhang, F.Q. (2012) The Evaluation of Intercuspal Occlusion of Healthy People with T-Scan II System. Shanghai Journal of Stomatology, 21, 62-65. (In Chinese)

[19] Kurita, H., Ikeda, K. and Kurashina, K. (2000) Evaluation of the Effect of a Stabilization Splint on Occlusal Force in Patients with Masticatory Muscle Disorders. Journal of Oral Rehabilitation, 27, 79-82.

[20] Canay, S., Cindaş, A., Uzun, G., et al. (1998) Effect of Muscle Relaxation Splint Therapy on the Electromyographic Activities of Masseter and Anterior Temporalis Muscles. Oral Surgery, Oral Medicine, Oral Pathology, Oral Radiology, and Endodontology, 85, 674-679.

[21] Huang, Z.S., Lin, X.F. and Li, X.L. (2011) Characteristics of Temporomandibular Joint Vibrations in Anterior Disk Displacement with Reduction in Adults. Cranio, 29, 276-283.

[22] Rosar, J.V., Barbosa, T.S., Dias, I.O.V., et al. (2017) Effect of Interocclusal Appliance on Bite Force, Sleep Quality, Salivary Cortisol Levels and Signs and Symptoms of Temporomandibular Dysfunction in Adults with Sleep Bruxism. Archives of Oral Biology, 82, 62-70. https://doi.org/10.1016/j.archoralbio.2017.05.018

[23] Harada, T., Ichiki, R., Tsukiyama, Y., et al. (2006) The Effect of Oral Splint Devices on Sleep Bruxism: A 6-Week Observation with an Ambulatory Electromyographic Recording Device. Journal of Oral Rehabilitation, 33, 482-488. https://doi.org/10.1111/j.1365-2842.2005.01576.x

[24] Karakis, D., Dogan, A. and Bek, B. (2014) Evaluation of the Effect of Two Different Occlusal Splints Onmaximum Occlusal Force in Patients with Sleep Bruxism: A Pilot Study. The Journal of Advanced Prosthodontics, 6, 103-108. https://doi.org/10.4047/jap.2014.6.2.103

[25] Clark, G.T., Beemsterboer, P.L., Solberg, W.K., et al. (1979) Nocturnal Electromyographic Evaluation of Myofascial Pain Dysfunction in Patients Undergoing Occlusal Splint Therapy. The Journal of the American Dental Association, 99, 607-611.

[26] Pierce, C.J. and Gale, E.N. (1988) A Comparison of Different Treatments for Nocturnal Bruxism. Journal of Dental Research, 67, 597-601.

[27] Dube, C., Rompre, P.H., Manzini, C., et al. (2004) Quantitative Polygraphic Controlled Study on Efficacy and Safety of Oral Splint Devices in Tooth-Grinding Subjects. Journal of Dental Research, 83, 398-403. https://doi.org/10.1177/154405910408300509 
[28] Holmgren, K., Sheikholeslam, A. and Riise, C. (1993) Effect of a Full-Arch Maxillary Occlusal Splint on Parafunctional Activity during Sleep in Patients with Nocturnal Bruxism and Signs and Symptoms of Cranio Mandibular Disorders. The Journal of Prosthetic Dentistry, 69, 293-297.

https://doi.org/10.1016/0022-3913(93)90109-2

[29] Yap, A.U. (1998) Effects of Stabilization Appliances on Nocturnal Parafunctional Activities in Patients with and without Signs of Temporomandibular Disorders. Journal of Oral Rehabilitation, 25, 64-68.

https://doi.org/10.1046/j.1365-2842.1998.00194.x 